\title{
Pheochromocytoma multisystem crisis treated with emergency surgery: a case report and literature review
}

\author{
Katsura Kakoki', Yasuyoshi Miyata ${ }^{1 *}$, Youhei Shida' ${ }^{1}$,Tomoaki Hakariya ${ }^{1}$, Kosuke Takehara', Seiya Izumida², \\ Motohiro Sekino ${ }^{3}$, Naoe Kinoshita ${ }^{4}$, Tsukasa Igawa $^{1,5}$, Junya Fukuoka ${ }^{4}$ and Hideki Sakai ${ }^{1}$
}

\begin{abstract}
Background: Pheochromocytoma is a neuroendocrine tumor that predominantly presents with hypertension, palpitations, and tachycardia due to excessive catecholamine excretion. Although pheochromocytoma multisystem crisis (PMC) is relatively rare, urologists and clinicians should focus on early diagnosis as delay in initiating the appropriate treatment can lead to mortality
\end{abstract}

Case presentation: A 70-year-old man developed ileus after a few days of medication for hypertension. Computed tomography incidentally revealed a left adrenal mass. This finding together with his clinical course was compatible with pheochromocytoma. An a-blocker was administered immediately, and his blood pressure was well controlled. However, his general condition and laboratory data deteriorated rapidly, and the patient was diagnosed with PMC with lethal status. Thus, emergency adrenalectomy was performed without confirmation of catecholamine levels. From the resected specimen, his tumor was judged as pheochromocytoma. On immunohistochemical analysis, the proliferation index evaluated by Ki-67 staining was $9.7 \%$. This case report was approved by the Human Ethics Review Committee of the Nagasaki University Hospital.

Conclusion: The present case of PMC was successfully treated with emergency surgery. The benign pheochromocytoma also presented with high cell proliferation potential, which may be a cause of the extreme aggressiveness of PMC.

Keywords: Pheochromocytoma multisystem crisis, Treatment strategy, Outcome, Cell proliferation, Malignant pheochromocytoma

\section{Background}

Pheochromocytoma is a relatively rare neuroendocrine tumor that predominantly presents with proximal or sustained hypertension, palpitations, tachycardia, and sweating due to excessive catecholamine release. These symptoms are often severe, and some patients can enter 'pheochromocytoma crisis' [1,2]. Patients judged to be in pheochromocytoma crisis require treatment with antihypertensive drugs and transfusion. The standard treatment for 'non-crisis' pheochromocytoma is generally

\footnotetext{
*Correspondence: int.doc.miya@m3.dion.ne.jp

${ }^{1}$ Department of Urology, Nagasaki University, Graduate School of Biomedical Sciences, 1-7-1 Sakamoto, Nagasaki 852-8501, Japan Full list of author information is available at the end of the article
}

preoperative preparation with an $\alpha$-blocker and surgical resection [1].

Pheochromocytoma multisystem crisis (PMC) is a fatal condition characterized by multiple organ failure, severe blood pressure variability, high fever, and encephalopathy [2]. Importantly, PMC is not synonymous with malignant hypertension caused by the massive release of catecholamine's from the pheochromocytoma. Indeed, several patients with PMC were reported to be normotensive or hypotensive [3, 4]. In addition, the symptoms and pathological conditions of PMC vary greatly. Abdominal pain, nausea, and dyspnea are common, while anemia, back pain, night sweat, and acidosis have also been reported [5]. Therefore, many patients with PMC are unrecognized 
at diagnosis [5]. The treatment strategy for patients with PMC requires particular attention, as delayed therapy can lead to severe sequelae or mortality.

Detailed information on the diagnosis and treatment of PMC is important for physicians. In addition, understanding the pathological characteristics of PMC is essential for developing diagnostic tools and treatment strategies. Unfortunately, there are few reports on the molecular biological findings or pathological characteristics in PMC patients [6]. Herein, we report a case of a patient with PMC who was successfully treated with emergency surgery. We also provide a literature review of the symptoms, data, treatments, and outcome of patients with PMC, as well as the relationship between cell proliferation and malignant potential in pheochromocytoma. The present case report presents new and important information for future studies on PMC. The authors obtained approval from the Human Ethics Review Committee of Nagasaki University Hospital for the publication of this report.

\section{Case presentation}

A 70-year-old man was admitted to our hospital for ileus and uncontrollable high blood pressure. His systolic blood pressure exceeded $160 \mathrm{mmHg}$, and oral diltiazem and amilodipin were initiated 5 days before the onset of ileus. Computed tomography showed a left adrenal mass and ileus (Fig. 1). His consciousness level was low with incoherent speech. His body temperature was $37.4{ }^{\circ} \mathrm{C}$, pulse rate was $126 \mathrm{bpm}$, blood pressure was $210 / 146 \mathrm{mmHg}$, and $\mathrm{SpO}^{2}$ was $98 \%$ on room air. He appeared to be sweating profusely, with cold moist peripheries. Electrocardiogram showed sinus rhythm tachycardia and short $\mathrm{PQ}$, high voltage, mitral $\mathrm{P}$, and

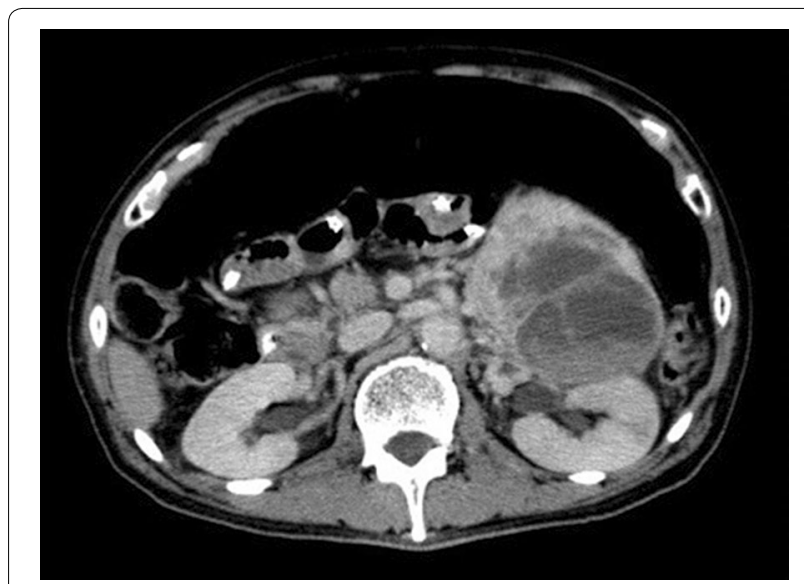

Fig. 1 Abdominal computed tomography revealed a large left adrenal mass with heterogeneous enhancement and extended intensity compatible with ileus negative $\mathrm{T}$ on V4-6. Abdominal radiography showed large intestine expansion due to gas. Initial laboratory data showed low thrombocyte count $(74,000 / \mu \mathrm{L})$, normal white blood cell count $(6000 / \mu \mathrm{L})$, high levels of hemoglobin $(16.3 \mathrm{~g} / \mathrm{dL})$, C-reactive protein $(9.30 \mathrm{mg} /$ $\mathrm{dL})$, serum glucose $(201 \mathrm{mg} / \mathrm{dL})$, and blood urea nitrogen $(48 \mathrm{mg} / \mathrm{dL})$, and normal level of serum creatinine $(1.05 \mathrm{mg} / \mathrm{dL})$. Amino-terminal pro-brain natriuretic peptide was extremely high at $13,938.0 \mathrm{ng} / \mathrm{mL}$.

We suspected pheochromocytoma on the basis of his computed tomography findings and clinical features. Doxazosin (2 mg/day orally), nicardipine $(1.5 \mu \mathrm{g} / \mathrm{kg} / \mathrm{h}$ i.v.), and landiolol (200 mg/day i.v.) were administered for blood pressure control. Because of the high fever, we suspected necrotizing enteritis due to ileus. Therefore, intravenous antibiotics were administered. Moreover, creatinine kinase and lactose dehydrogenase increased rapidly to 7066 and $609 \mathrm{IU} / \mathrm{L}$, respectively. At that time, his body temperature was $40.1{ }^{\circ} \mathrm{C}$. His respiratory state was aggravated after 3 days in the hospital, and oxygen was started. Laboratory tests showed that renal and liver functions were deteriorating. Five days after hospitalization, tracheal intubation was performed in the intensive care unit. Artificial ventilation and continuous hemodiafiltration were started.

The patient was diagnosed with PMC on the basis of the clinical course and radiological findings, without considering serum catecholamine levels. We performed emergency left adrenalectomy despite his critical condition, as he showed poor control and was refractory to medical treatment. Intraoperatively, the anesthesiologist used several types of antihypertensive drugs including doxazosin, landiolol hydrochloride, and diltiazem as necessary. Although temporary blood pressure was reduced to $80 / 46 \mathrm{mmHg}$ after removal of the adrenal gland, the patient's vital signs stabilized quickly after catecholamine infusion.

Postoperatively, the patient's blood catecholamine levels intraoperatively were: epinephrine 10,351 (normal range $\leq 100 \mathrm{ng} / \mathrm{mL}$ ), norepinephrine $27,654 \mathrm{ng} / \mathrm{mL}$ (normal range $100-450 \mathrm{ng} / \mathrm{mL}$ ), and dopamine 209,500 ng/ $\mathrm{mL}$ (normal range $\leq 20 \mathrm{ng} / \mathrm{mL}$ ). The patient recovered rapidly postoperatively. One day postoperatively, his body temperature normalized. Furthermore, lactose dehydrogenase, creatinine kinase, liver function, respiratory status, and consciousness began to improve. He was weaned from ventilatory support at 2 days postoperatively, and from continuous hemodiafiltration and catecholamine administration at 3 days postoperatively. $\mathrm{He}$ was discharged 37 days postoperatively. Various imaging examinations including computed tomography and magnetic resonance imaging were performed during 
hospitalization to reconfirm the absence of metastatic mass. He has had no tumor recurrence or metastasis for 2 years.

The resected tumor was $12 \times 11 \times 8 \mathrm{~cm}$ in size and weighed $530 \mathrm{~g}$, with prominent central necrosis and internal bleeding (Fig. 2). Histologically, the tumor had a capsule and was comprised of basophilic granular cells with polygonal features (Fig. 3). On immunohistochemistry, the specimen stained positive for chromogranin A, NCAM, and S100. Therefore, the tumor was identified as a pheochromocytoma. The cell proliferation index measured using an anti-Ki-67 antibody was 9.7 \% (Fig. 4).

\section{Discussion}

The incidence of pheochromocytoma is approximately $0.1 \%$ in the hypertensive population [7]. Pheochromocytoma typically arises from the adrenal medulla, although may develop in chromaffin cells in or around sympathetic ganglia [8]. The standard treatment for symptomatic pheochromocytoma is generally surgical resection. $\mathrm{PMC}$ is a more severe and potentially lethal condition of pheochromocytoma, and represents an endocrinological emergency [9]. Therefore, clinicians should have detailed information regarding the symptoms, diagnosis, and treatment of PMC, despite its rarity.

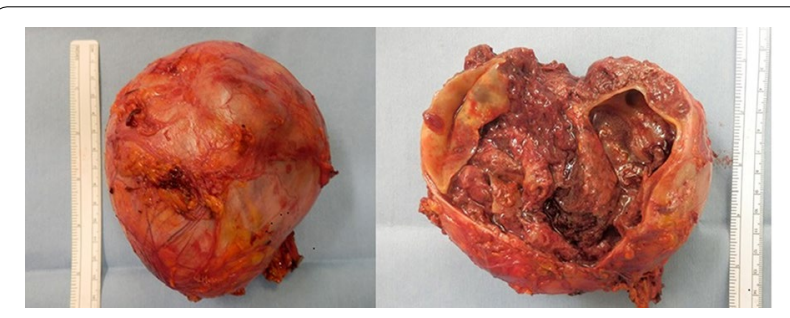

Fig. 2 Resected specimen showing a tumor measuring $14 \mathrm{~cm}$ in diameter. There was hemorrhage and necrosis within the tumor

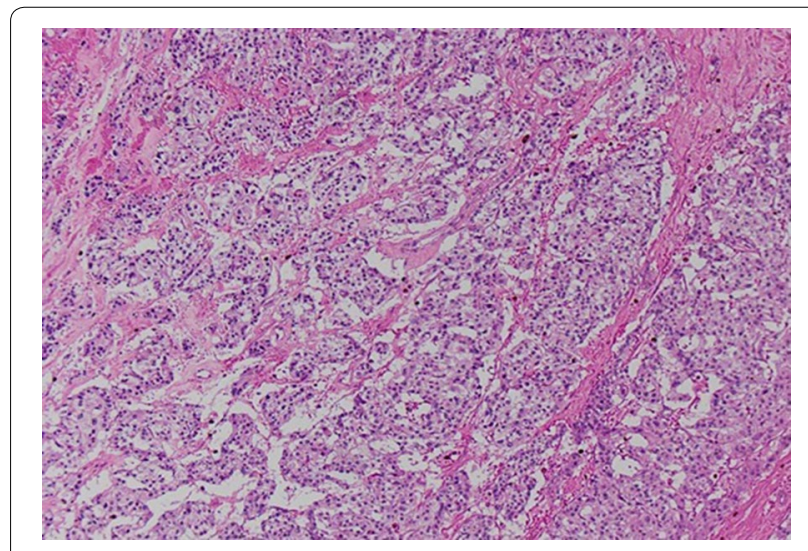

Fig. 3 Hematoxylin-eosin staining (magnification: $\times 40$ )

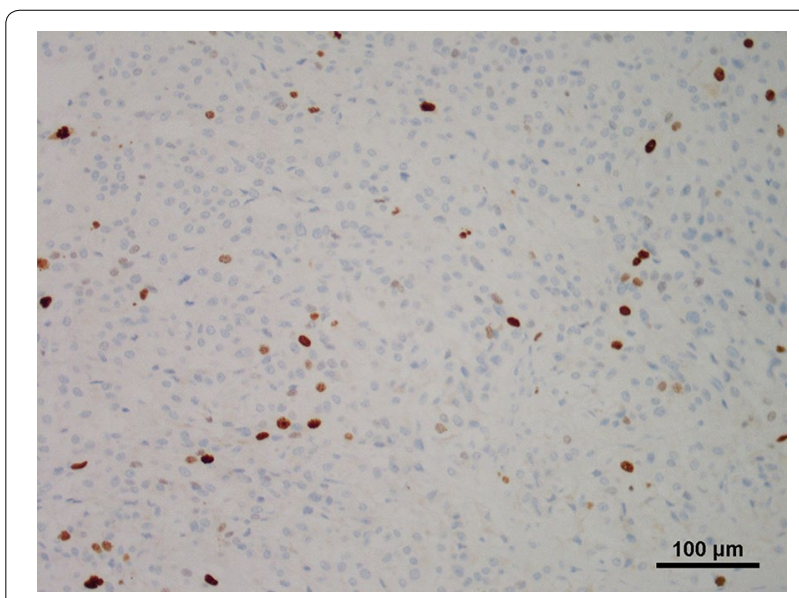

Fig. 4 Ki-67 staining. The distribution of Ki-67-stained cells was nonspecific (magnification: $\times 200$ )

Several previous reports have stressed the importance of emergency treatment for PMC to avoid death [2, 6]. Indeed, all European cases of PMC without surgical resection $(5 / 12,41.7 \%)$ died despite conservative treatment (Table 1). The results of physical examination and hormonal studies in previous PMC cases are shown in Table 1. Although extremely high blood pressure and fever are characteristics of PMC, two patients had no high-grade fever. Other signs and symptoms of PMC have been reported [5]. Therefore, accurate diagnosis on the basis of vital signs, symptoms, and physical examination can be difficult.

Our patient presented with hypertension and ileus at onset, while subsequent computed tomography performed to examine the ileus revealed a left adrenal tumor. On admission, the patient did not present with high fever but showed a slight disturbance of consciousness and extremely high blood pressure. Despite blood pressure control with $\alpha 1$-blocker treatment after hospitalization, his body temperature increased and consciousness worsened. Moreover, liver dysfunction and respiratory distress developed. Thus, his symptoms met the diagnostic criteria of PMC. On the basis of these findings, we opted for emergency surgery before confirmation of blood catecholamine levels and Methoxy-Isobutyl-Isonitrile (MIBI) scintigraphy. His condition improved dramatically after surgery. He was weaned from continuous hemodiafiltration and respiration, catecholamine administration, and intubation at 4 days postoperatively.

Our case provides further support that surgical resection is important for preventing mortality in patients with PMC. Nevertheless, the appropriate timing of surgery remains unclear. As shown in Table 1, surgery on the day following admission may not be associated with outcome $[2,10,11]$. 
Table 1 Characteristics, physical conditions, treatments, and outcomes of patients with pheochromocytoma multisystem crisis

\begin{tabular}{|c|c|c|c|c|c|c|c|c|c|c|}
\hline Sex & $\begin{array}{l}\text { Age } \\
\text { (years) }\end{array}$ & $\begin{array}{l}\text { S/DBP } \\
(\mathrm{mmHg})\end{array}$ & $\mathrm{BT}\left({ }^{\circ} \mathrm{C}\right)$ & $\begin{array}{l}\text { Size } \\
(\mathrm{cm})\end{array}$ & $\begin{array}{l}\text { Ad } \\
\text { (Times }\end{array}$ & $\begin{array}{l}\text { NAd } \\
\text { pper lir }\end{array}$ & Dopa & $\begin{array}{l}\text { Operation } \\
\text { (days) }^{\mathbf{b}}\end{array}$ & Outcome & Year [Ref] \\
\hline $\mathrm{F}$ & 53 & 210/110 & 40 & 6.3 & 101.1 & 103.5 & - & Not performed & Died & 1988 [2] \\
\hline$F$ & 62 & $285 / 140$ & 40.5 & 10 & 96.0 & 14.8 & - & Emergency & Survived & 1988 [22] \\
\hline $\mathrm{F}$ & 50 & $200 / 90$ & 40 & 7 & 43.3 & 23.4 & - & 7 & Survived & 1988 [2] \\
\hline $\mathrm{F}$ & 65 & $120 / 80$ & 39.5 & 4 & - & - & - & $>21$ & Survived & 1993 [10] \\
\hline$M$ & 31 & $164 / 40$ & 41.0 & - & $10^{\mathrm{a}}$ & $6.7^{a}$ & $1.3^{\mathrm{a}}$ & 36 & Survived & $1997[11]$ \\
\hline$F$ & 50 & $160 / 100$ & 41.5 & - & - & - & - & Not performed & Died & $2002[12]$ \\
\hline $\mathrm{F}$ & 41 & 190/130 & 41.0 & - & - & - & - & - & Survived & 2006 [9] \\
\hline $\mathrm{F}$ & 26 & $160 / 110$ & - & 4.5 & - & - & - & Not performed & Died & 2008 [6] \\
\hline$M$ & 39 & $240 / 140$ & - & 5.5 & 41.0 & 49.6 & - & Emergency & Survived & 2008 [6] \\
\hline $\mathrm{F}$ & 52 & $300 / 200$ & 39.9 & 10 & - & - & - & Not performed & Died & 2010 [13] \\
\hline $\mathrm{F}$ & 52 & $162 / 104$ & 35.8 & - & 103.5 & 53.9 & 53.1 & - & Survived & 2010 [5] \\
\hline$M$ & 27 & $160 / 120$ & - & 5 & - & - & - & Not performed & Died & $2012[14]$ \\
\hline$M$ & 70 & $210 / 146$ & 40.1 & 5 & 1047.5 & 61.5 & 103.5 & 5 & Survived & Present \\
\hline
\end{tabular}

$M$ male, $F$ female, $S / D B P$ systolic/diastolic blood pressure, $B T$ body temperature, $A d$ adrenalin, NAd noradrenalin, Dopa dopamine, Ref reference number

a 24 h urine correction

b Operation day following admission

In addition, other vital signs and clinicopathological features do not appear to be associated with outcome [2, 5, 6, 9-14]. Thus, in addition to the difficulty in diagnosis, the optimal treatment strategies for patients with $\mathrm{PMC}$ remain unclear.

The proliferation index evaluated by Ki-67 is commonly used to assess the cell-cycle. A number of case series have reported a PI measured by Ki-67 staining of less than $1 \%$ in benign pheochromocytoma $[15,16]$ (Table 2). By contrast, our case of benign pheochromocytoma showed an extremely high level of cell proliferation potential, with a PI of $9.7 \%$. The relationships between cell proliferation and pathological characteristics including hormone secretion, tumor size, and symptoms in benign pheochromocytoma are not fully understood, although PI was reported to be significantly associated with capsule invasion of pheochromocytoma [24]. The relationship between the aggressiveness of PMC and cell proliferation is unknown, as this is the first report on cell proliferation in PMC. Speculatively, the high proliferative potential despite benign tumor status may be a characteristic of PMC that contributes to its aggressive characteristics and severe symptoms in patients.

PI is also a useful marker for distinguishing benign from malignant pheochromocytoma [16-19]. We identified nine studies in PubMed describing a PI cut-off in adrenal malignant pheochromocytoma tissues; the suggested cut-offs for predicting malignant behavior are shown in Table 2 . The PI in the present case was relatively higher

Table 2 Mean proliferation indexes of benign and malignant adrenal pheochromocytoma

\begin{tabular}{|c|c|c|c|c|c|c|c|c|c|}
\hline \multicolumn{10}{|l|}{ Benign } \\
\hline $\mathrm{N}$ & $23^{b}$ & 16 & 93 & 21 & 20 & - & 41 & 44 & 14 \\
\hline PI (\%) & 1 & 0.75 & 0.21 & 0.9 & $<1$ & - & 0.8 & $<1$ & 2.2 \\
\hline \multicolumn{10}{|l|}{ Malignant } \\
\hline$N$ & 10 & 15 & 36 & 4 & $3^{c}$ & 43 & 5 & 11 & 7 \\
\hline PI (\%) & 5 & 13.9 & 3.0 & 4.3 & $<1$ & 7.8 & 1.4 & - & 14.1 \\
\hline Cut-offa & $>3$ & $\geq 3$ & $>2.5$ & - & - & $>5$ & $>2$ & $>5$ & - \\
\hline Year & 1998 & 2000 & 2000 & 2001 & 2003 & 2004 & 2008 & 2010 & 2012 \\
\hline Reference & [17] & [18] & [19] & {$[20]$} & [15] & {$[21]$} & {$[22]$} & {$[16]$} & {$[23]$} \\
\hline
\end{tabular}

PI proliferation index

a Cut-off of $\mathrm{PI}(\%)$ between benign and malignant pheochromocytoma

b Including extra-adrenal tumors

c Patients without metastasis at diagnosis 
than the mean PI in malignant pheochromocytoma, indicating malignant and metastatic potential. However, no recurrence or metastatic mass was detected at 2 years postoperatively. Nevertheless, follow-up with imaging and hormonal studies are required as a part of the pheochromocytoma can recur in various organ types [25].

\section{Conclusions}

We report a case of rapidly progressing and life-threatening PMC. Although it is generally difficult to decide on the surgical strategy for patients with PMC, emergency surgery is important for achieving a favorable outcome. Therefore, urologists and clinicians should be aware of this rare life-threatening condition. In addition, we speculate that the aggressive characteristics of PMC may be due to a high proliferative potential. Additional studies examining the pathological characteristics of the disease at the molecular level are required to determine the biological characteristics of PMC and to develop strategies for diagnosis and treatment.

\section{Consent}

Written informed consent was obtained from the patient for the publication of this Case Report and any accompanying images.

\section{Abbreviations \\ PMC: pheochromocytoma multisystem crisis; PI: proliferation index; MIBI: methoxy-Isobutyl-Isonitrile.}

\section{Authors' contributions}

YM and HS were responsible for the concept, design, and drafting of the manuscript. YM obtained approval from the institutional ethical review board. KK, YS, TH, SI, MS, JF, and KN were responsible for acquisition of the patients' data. KT and TI critically reviewed and amended the manuscript for important intellectual content. All authors read and approved the final manuscript.

\section{Author details}

${ }^{1}$ Department of Urology, Nagasaki University, Graduate School of Biomedical Sciences, 1-7-1 Sakamoto, Nagasaki 852-8501, Japan. ${ }^{2}$ Department of Cardiovascular Medicine, Nagasaki University Graduate School of Biomedical Sciences, Nagasaki 852-8501, Japan. ${ }^{3}$ Division of Intensive Care, Nagasaki University Hospital, Nagasaki 852-8501, Japan. ${ }^{4}$ Department of Pathology, Nagasaki University Hospital, Nagasaki 852-8501, Japan. ${ }^{5}$ Department of Urology, Kurume University Hospital, Fukuoka 830-0011, Japan.

\section{Acknowledgements}

The authors thank Mr. Takumi Shimogama for his outstanding support. This work was not supported by any funding.

\section{Competing interests}

The authors declare that they have no competing interests.

Received: 8 June 2015 Accepted: 24 November 2015

Published online: 09 December 2015

\section{References}

1. Whitelaw BC, Prague JK, Mustafa OG, Schulte KM, Hopkins PA, Gilbert JA, et al. Pheochromocytoma crisis. Clin Endocrinol. 2014;80:13-22.
2. Newell K, Prinz RA, Braithwaite S, Brooks M. Phaeochromocytoma crisis. Am J Hypertens. 1988;1:189s-91s.

3. Herbland A, Bui N, Rullier A, Vargas F, Gruson D, Hilbert G. Multiple organ failure as initial presentation of pheochromytoma. Am J Emerg Med. 2005;23:565-6.

4. van Lennep JR, Romijn JA, Harinck HI. Multi-organ failure after a glucagon test. Lancet. 2007;369:798.

5. Uchida N, Ishiguro K, Suda K, Nishimura M. Pheochromocytoma multisystem crisis successfully treated by emergency surgery: report of case. Surg Today. 2010:40:990-6.

6. Rosas AL, Kasperlik-Zaluska AA, Eisenhofer G, Bass BL, Pacak K, Eisenhofer G. Pheochromocytoma crisis induced by glucocorticoids: a report of four cases and review of literature. Eur J Endocrinol. 2008;158:423-9.

7. Benowitz NL. Pheochromocytoma-recent advances in diagnosis and treatment. West J Med. 1988;148:561-7.

8. Bravo EL, Gifford RW Jr. Pheochromocytoma. Endocrinol Metab Clin North Am. 1993:22:329-41.

9. Moran ME, Rosenberg DJ, Zornow DH. Pheochromocytoma multisystem crisis. Urology. 2006;67:864.e19-846.e20.

10. Lorz W, Cottier C, Imhof E, Gyr N. Multiple organ failure and coma as initial presentation of pheochromocytoma in a patient with multiple endocrine neoplasia (MEN) type II A. Intensive Care Med. 1993;19:235-8.

11. Salahuddin A, Rohr-Kirchgraber T, Shekar R, West B, Loewenstein J. Interleukin-6 in the fever and multiorgan crisis of pheochromocytoma. Scand J Infect Dis. 1997;29:640-2.

12. Caputo C, Fishbane S, Shapiro L, Courgi RG, Kostadinov S, Donovan V, et al. Pheochromocytoma multisystem crisis in a patient with multiple endocrine neoplasia type IIB and pyelonephritis. Am J Kidney Dis. 2002;39:E23.

13. Davlouros PA, Velissaris D, Tsilola A, Filos KS, Alexopoulos D. Fever with multiple organ failure: not always sepsis. Aneasth Intensive Care. 2010;38:1090-3.

14. Gundgurthi A, Gupta S, Garg MK, Granguly P, Bhardwaj R. Unsuspected pheochromocytoma multisystem crisis: a fatal outcome in a young male patient. J Assoc Physicians India. 2012;60:53-60.

15. Elder EE, Xu D, Höög A, Enberg U, Hou M, Pisa P, et al. Kl-67 AND hTERT expression can aid in the distinction between malignant and benign pheochromocytoma and paraganglioma. Mod Pathol. 2003;16:246-55.

16. Tavangar SM, Shojaee A, Moradi Tabriz H, Haghpanah V, Larijani B, Heshmat R, et al. Immunohistochemical expression of Ki67, c-erbB-2, and c-kit antigens in benign and malignant pheochromocytoma. Pathol Res Pract. 2010;206:305-9.

17. Clarke MR, Weyant J, Watson CG, Carty AS. Prognostic markers in phenochromovytoma. Hum Pathol. 1998;29:522-6.

18. Gupta D, Shidham V, Holden J, Layfield L. Prognostic value of immunohistochemical expression of topoisomerase alpha II, MIB-1, p53, E-cadherin, retinoblastoma gene protein product, and HER-2/neu in adrenal and extra-adrenal pheochromocytomas. Appl Immunohistochem Mol Morphol. 2000;8:267-74.

19. der Harst Van, Bruining HA, Jaap Bonjer H, van der Ham F, Dinjens WN, Lamberts SW, et al. Proliferative index in pheochromocytomas; does it predict the occurrence of metastasis. J Pathol. 2000;19:175-80.

20. Ohji H, Sasagawa I, Ichiyanagi O, Suzuki Y, Nakada T. Tumour angiogenesis and Ki-67 expression in phenochromocytoma. BJU Int. 2001;87:381-5.

21. August C, August K, Schroeder S, Bahn H, Hinze R, Baba HA, et al. CGH and CD 44/MIB-1 immunohistochemistry are helpful to distinguish metastasized from nonmetastasized sporadic pheochromocytomas. Mod Pathol. 2004;17:1119-28.

22. Strong VE, Kennedy T, Al-Ahmadie H, Tang L, Coleman J, Fong Y, et al. Prognostic indicators of malignancy in adrenal pheochromocytomas: clinical, histopathologic, and cell cycle/apoptosis gene expression analysis. Surgery. 2008;143:759-68.

23. de Wailly P, Oragano L, Radé F, Beaulieu A, Arnault V, Levillain P, et al. Malignant phenochromocytoma: new malignancy criteria. Langenbecks Arch Surg. 2012;397:239-46.

24. Ocal I, Avci A, Cakalagaoglu F, Can H. Lack of correlations among histopathological parameters, Ki-67 proliferation index and prognosis in pheochromocytoma patients. Asian Pac J Cancer Prev. 2014;15:1751-5.

25. Beilan JA, Lawton A, Hajdenberg J, Rosser CJ. Pheochromocytoma of the urinary bladder: a systematic review of the contemporary literature. BMC Urol. 2013;13:22. 